\title{
RDUS
}

Revue de DROIT UNIVERSITÉ DE SHERBROOKE

Titre : $\quad$ LE TRIPTYQUE SANTÉ - IDENTITÉS AUTOCHTONES - TERRITOIRE. PERSPECTIVES JURIDIQUES

Auteur(s) : $\quad$ Doris FARGET

Revue : $\quad$ RDUS, 2009-2010, volume 40, numéro 1-2

Pages: $\quad 97-130$

ISSN : $\quad 0317-9656$

Éditeur : $\quad$ Université de Sherbrooke. Faculté de droit.

URI : $\quad$ http://hdl.handle.net/11143/10383

DOI : https://doi.org/10.17118/11143/10383 
Page vide laissée intentionnellement. 


\title{
ARTICLE
}

\section{LE TRIPTYQUE SANTÉ - IDENTITÉS AUTOCHTONES - TERRITOIRE. PERSPECTIVES JURIDIQUES}

\begin{abstract}
par Doris FARGET*
L'objectif de cet article est de mettre en valeur la relation qui existe entre les identités autochtones, la santé et les droits au territoire. Nous nous concentrons sur la circonscription du risque en santé, son intensité et sa globalité, afin d'en situer les sources, celles-ci étant multiples mais provenant initialement de la colonisation. Dans ce contexte, nous postulons que le droit est un espace qui reflète les valeurs sociopolitiques dominantes. De ce fait, le risque en santé chez les membres des Premières nations est entretenu par l'ordonnancement juridique, en raison de l'inadaptation à la diversité des situations de ce dernier et des décalages par rapport au contexte autochtone qu'il reproduit. Il existe donc des répercussions juridiques sur ce risque. Celles-ci proviennent, d'une part, de l'absence d'accès sécurisé aux territoires ancestraux et, d'autre part, des décalages dans l'élaboration et la mise en ouvre du droit.

The purpose of this article is to highlight the relationship between the identity of indigenous peoples, their health considerations and their right to claim traditional lands. This article seeks to circumscribe the health risk associated with a lack of access to ancestral lands by First Nations. The strength of that risk and its prevalence as a means of identifying its primary source - colonialism - are examined. In this context, the writer postulates that the law is a reflection of the dominant socio-political values. As a result, the health risk run by members of First Nations is fostered by the law because of its inability to adapt to the situational diversity and needs of indigenous peoples. Thus, the legal consequences of this risk arise from the lack of secure access to ancestral lands and from inconsistencies between the elaboration and the implementation of the law.
\end{abstract}

* Doctorante en cotutelle à la Faculté de droit de l'Université de Montréal et à la Faculté de droit de l'Université Aix-Marseille 3. Elle est également assistante de recherche au Centre de recherche en droit public de la Faculté de droit de l'Université de Montréal et membre associée au Centre en droit international du développement durable de l'Université McGill. L'auteure tient à remercier Emmanuelle Bédard, professeur à l'Université de Rimouski, Michèle Audette ainsi que son équipe de Nouveaux sentiers: Fondation pour les Premières nations, Karine Gentelet, Christine Vézina et Emmanuelle Bernheim pour leur relecture attentive et leurs commentaires, de même que l'ensemble des participants au colloque Risque et populations marginalisées: une perspective de santé, qui s'est tenu dans le cadre du Congrès de l'ACFAS, le 13 mai 2009, à l'Université d'Ottawa. 


\section{SOMMAIRE}

Introduction

I. Circonscription du risque en santé et de ses sources, chez les peuples autochtones au Québec ..... 101

L'intensité du risque .............................................. 104

Deux niveaux de risques et une source principale......... 106

Globalité $d u$ risque .......................................... 109

II. Le droit et le risque en santé chez les peuples autochtones................................................... 110

Non reconnaissance en droit interne d'un accès sécurisé aux territoires ancestraux .......................... 112 Entretien du risque en santé par d'autres mécanismes juridiques 
Le triptyque santé

(2009-10) 40 R.D.U.S. - identités autochtones -

99

territoire. Perspectives juridiques

\section{Introduction}

L'objectif de cet article est de circonscrire ce qu'est le risque en santé en contexte autochtone, puis de mettre en évidence la relation qu'il entretient avec l'identité et le territoire, pour enfin souligner l'impact que peut avoir le droit dans la reproduction de ce risque ${ }^{1}$.

Afin d'aborder ce rapport, il nous faut rappeler une tension principale. Il s'agit de celle qui confronte les dynamiques de marginalisation et de prise de pouvoir des Autochtones. La première implique que nous ayons conscience des décalages et des échecs propres aux sociétés contemporaines en matière d'intégration. La seconde suppose, parallèlement, que nous gardions à l'esprit ce que représentent les Autochtones aujourd'hui, à savoir des agents de pouvoir désireux et aptes à transformer la société. Ainsi, aucun acteur ne dispose d'un rôle providentiel. L'État ne doit pas être considéré comme l'agent unique de changement, ce qui irait dans le sens de la reproduction des décalages actuels ainsi que d'un paternalisme accru. Par ailleurs, l'illusion de liberté des Autochtones, en tant qu'agents de pouvoir, disposés à impulser et créer seuls la transformation ne doit pas non plus être entretenue. C'est pourquoi, le contexte favorable à la transformation se situe au sein d'un équilibre entre auto-affirmation, écoute et interventionnisme étatique. Le point de vue que nous allons présenter, bien que mettant fortement l'accent sur la situation de marginalisation en raison du sujet abordé, se situe, toutefois, au sein de cette tension.

Les peuples autochtones ont une relation spécifique et privilégiée avec leurs terres ancestrales. Le territoire est un espace important dans le vécu de leurs identités. Bien qu'il ne soit pas l'élément unique de ce vécu, puisque l'identité implique aussi une relation particulière au temps et aux autres, des valeurs propres ainsi qu'un sentiment d'appartenance, la relation au territoire

1. Nous limitons l'examen de cette équation aux peuples autochtones du Québec, parce qu'elle est particulièrement bien documentée. 
demeure être un élément central ${ }^{2}$. En contexte autochtone, il représente un lieu d'expression, de pratiques et de transmission des identités et sa connaissance est importante, notamment en matière de spiritualité et de bien être ${ }^{3}$. Jean-Paul Lacasse, citant un interlocuteur autochtone, indique par exemple que "[...] l'ordre innu se rapportant à l'appartenance des Innus à la terre provient de leur relation unique avec la terre, leur mère: 'Nous appartenons à la terre; la terre et nous ne sommes qu'un corps et un esprit. La terre, c'est notre vie' "4. D'où l'importance de considérer cette relation.

Qu'entend-t-on par identités autochtones et quel est le lien avec la santé? L'Enquête régionale longitudinale sur la santé des Premières Nations de la région du Québec indique que :

L'identité peut se définir comme étant la conscience qu'un individu a par rapport à son appartenance à un groupe

2. Karine Gentelet, Alain Bissonnette et Guy Rocher, La sédentarisation : effets et suites chez des Innus et des Atikamekw, Montréal, Thémis, 2007, aux pp. 21-25 [Gentelet, Bissonnette et Rocher].

3. "Chez les Innus, la façon de voir le monde est globalisante et unifiée. [...] L'univers innu est communautaire et l'ordre existe en fonction du mieuxêtre du groupe. Vus sous cet angle, la propriété, le contrat et le châtiment n'ont pas de sens ou acquièrent un sens très différent. Pour Robert Vachon (1992: 6), l'Occidental a une perception anthropocentrique du monde, en ce sens que l'homme ou la femme a plus d'importance que la nature, alors que la tradition autochtone est cosmocentrique, en ce sens qu'elle est plus centrée sur l'univers et la nature auxquels appartient l'homme. C'est peut être en ce référant au lien qu'a l'Innu avec la Terre que l'ont peut le mieux saisir cette vision du monde. [...] les Innus entretiennent avec la terre et la faune des liens tels qu'ils ne peuvent se dire individuellement propriétaires de l'une ou de l'autre au sens où la société québécoise l'entend. [...] Alors que la société majoritaire conçoit le territoire et son utilisation en termes de propriété étatique ou individuelle, la société innue s'y réfère selon une approche de gestion des ressources au profit de la famille et de la communauté en général : il n'y a d'ailleurs pas de mot, dans la langue innu, pour désigner le concept de propriété au sens du Code civil. Mais les Innus considèrent néanmoins qu'ils ont un "titre " collectif de gestion sur le territoire", Jean-Paul Lacasse, Les Innus et le territoire, Québec, Septentrion, 2004, à la p. 37 [Lacasse].

4. Armand McKenzie, verbatim, mars 1992, cité dans Lacasse, supra note 3 à la p. 39. 
Le triptyque santé

- identités autochtones -

101

territoire. Perspectives juridiques

qui possède des caractéristiques distinctives (langue, valeurs, croyances, pratiques, etc.). Les problèmes identitaires sont un incontournable en ce qui a trait à la santé et au bien-être des autochtones ${ }^{5}$.

Par conséquent, santé, identité et territoire sont interreliés, d'où l'importance de les considérer ensemble. Formant un tout, la rupture ou l'atteinte portée à la relation au territoire ou à l'identité ont des conséquences sur la santé des membres des Premières nations ${ }^{6}$.

Afin d'exposer cette relation, nous allons d'abord circonscrire le risque en santé chez les peuples autochtones du Québec (I.). Ensuite, nous expliquerons comment le droit entretient ce risque (II.).

\section{Circonscription du risque en santé et de ses sources, chez les peuples autochtones au Québec}

Afin d'éclaircir notre propos et de poser le contexte, ce paragraphe a pour fonction d'illustrer et de circonscrire ce qu'est le risque en santé chez les peuples autochtones du Québec. Pour ce faire, nous allons nous appuyer sur certaines parties de l'Enquête régionale longitudinale sur la santé des Premières Nations de la région du Québec, diligentée par la Commission de la santé et des services sociaux des Premières nations du Québec et du

5. "Enquête régionale longitudinale sur la santé des Premières Nations de la région du Québec ", rapport sur l'influence du milieu sur la santé des Premières nations (2002), en ligne: Commission de la santé et des services sociaux des Premières nations du Québec et du Labrador [C.S.S.S.P.N.Q.L.] <http://www.cssspnql.com/fr/recherche/ documents/ ERS2002-Influencedumilieu.pdf $>$ à la p. 35.

6. "La perte d'identité peut, entre autres, contribuer au développement de différents problèmes psychosociaux. À ce sujet, les travaux de Chandler et Lalonde (1998) en Colombie-Britannique ont démontré que les communautés des Premières nations qui ont entrepris concrètement de préserver et de réhabiliter leur culture sont aussi celles qui ont les taux de suicide les plus bas. [...] Tout comme la langue, la culture et la spiritualité sont au cœur de la définition de l'identité d'un peuple ", Ibid. aux pp. 35-36. 
Labrador (la C.S.S.S.P.N.Q.L.)7 ${ }^{7}$ Plusieurs indicateurs de risque en santé, qui s'inscrivent au sein d'un contexte et de déterminants sociaux, sont mis en évidence par celle-ci.

Le premier indicateur réside dans la présence accrue des suicides. En effet, les peuples autochtones du Québec sont cinq fois plus exposés au suicide que les Québécois ${ }^{8}$. Le second indicateur touche au logement. La C.S.S.S.P.N.Q.L. indique sur ce point que 4200 foyers des Premières nations du Québec sur 12500 sont en situation de surpeuplement ${ }^{9}$. Le troisième

7. L'équipe de recherche de la C.S.S.S.P.N.Q.L. a travaillé au sein de 23 communautés et trois villes au Québec. Elle a interviewé 4000 personnes (des enfants, des adolescents et des adultes) pour parvenir à ses résultats. Il s'agit donc d'une enquête fiable, scientifiquement valide, menée par un organisme indépendant de l'État et par des chercheurs autochtones connaisseurs des communautés participantes et des problèmes sanitaires auxquels elles sont confrontées. Il s'agit enfin d'une étude longitudinale (entre 1997 et 2014) apte à dégager des tendances continues, puisqu'elle se déroule sur plusieurs années. C'est pourquoi nous nous appuyons sur ses données. Enfin, précisons que ses résultats sont confirmés par d'autres travaux, voir notamment Karine Gentelet, Gentelet, Bissonnette et Rocher, supra note 2.

8. Dévoilement d'un plan pour s'attaquer aux problèmes sociaux et de santé, en ligne: C.S.S.S.P.N.Q.L. <http://www.cssspnql.com/fr/nouvelles/ plan2007-2017.htm>. Voir aussi: "59,4\% des répondants hommes et $51,6 \%$ des répondantes femmes vivant hors communauté en milieu urbain ont déjà pensé au suicide dans leur vie, ce qui représente des écarts considérables par rapport aux proportions observées dans les communautés $(38,7 \%$ des hommes et 39,2 \% des femmes). Les jeunes adultes y ont déjà pensé en plus grand nombre, soit 62,3\% des 18 à 34 ans (50,5\% dans les communautés) et 48,6\% des 35 à 54 ans (38,9\% dans les communautés) ", "Enquête régionale longitudinale sur la santé des Premières Nations de la région du Québec ", rapport des Premières nations vivants hors communautés en milieu urbain (2002), en ligne : C.S.S.S.P.N.Q.L. <http://www.cssspnql.com/fr/recherche/documents/ ERS2002-Hors-communaute.pdf>, à la p. 71 .

9. "Plan directeur de la santé et des services sociaux des Premières Nations du Québec 2007-2017", en ligne: C.S.S.S.P.N.Q.L. <http://www. cssspnql.com/fr/nouvelles/documents/Plan\%20directeur.pdf $>$ à la p. 61. Voir aussi : "L'accessibilité à un logement adéquat est un problème répandu au sein de plusieurs communautés des Premières nations du Québec et du Canada. Parmi les obstacles à l'accès à un logement décent, mentionnons que le revenu moyen dans les communautés des 
$\begin{array}{cc}\text { (2009-10) } 40 \text { R.D.U.S. } & \text { Le triptyque santé } \\ \text { - identités autochtones - } \\ \text { territoire. Perspectives juridiques }\end{array}$

indicateur porte sur l'accès aux soins et services de santé. D'une part, "Seulement 18,8 \% des communautés offrent l'accès quotidien à un médecin et la moitié des communautés offrent l'accès à un médecin une fois par semaine "10. D'autre part, en termes d'accès et de pratique de la médecine traditionnelle, la Commission indique que "[p]rès du tiers des communautés $(31,3$ $\%$ offrent des services de médecine traditionnelle qui sont accessibles par le biais du centre de santé ou d'un programme communautaire "11. De plus, seule une communauté au Québec dispose d'un hôpital, les autres n'ont ni hôpital ni centre local de services communautaires. Le quatrième indicateur porte sur la pauvreté. L'enquête révèle, en la matière, qu'un membre des Premières nations sur cinq vit sous le seuil de pauvreté et qu'un peu moins de la moitié des adultes sont sans emploi dans les communautés des Premières nations. Elle rappelle à cet égard que la pauvreté est un déterminant de la santé et du bien-être ${ }^{12}$. Le

Premières nations est inférieur à la moitié de la moyenne canadienne. Ensuite, le taux de natalité y est deux fois plus élevé que la moyenne canadienne. Le MAINC estime que dans la prochaine décennie, on pourra compter une augmentation moyenne de 4500 nouveaux ménages [autochtones] par année dans l'ensemble du pays (45 000 nouveaux ménages en 10 ans). Ainsi, une incapacité à loger convenablement les ménages peut ultimement résulter en des problèmes de surpeuplement dans les logements existants ", "Enquête régionale longitudinale sur la santé des Premières Nations de la région du Québec ", supra note 5 à la p. 14.

10. "Plan directeur de la santé et des services sociaux des Premières Nations du Québec 2007-1017 ",ibid. à la p. iv.

11. Ibid.

12. "Enquête régionale longitudinale sur la santé des Premières Nations de la région du Québec ", rapport des Premières nations vivants dans les communautés (2002), en ligne : C.S.S.S.P.N.Q.L. <http://www.cssspnql. $\mathrm{com} / \mathrm{fr} /$ recherche/documents/ERS2002-Surcommunaute.pdf $>$ à la p. 203. Sur les répercussions de la pauvreté en santé, voir les travaux de la Commission macroéconomie et la santé, en ligne: World Health Organization <http://www3.who.int/ whosis/cmh/cmh_report/e/report. cfm>; voir aussi "The world health report 2002 - Reducing Risks, Promoting Healthy Life ", en ligne : Organisation mondiale de la santé (O.M.S.) <http://www. who.int/whr/2002/ chapter4/en/index1.html>; Voir enfin Secrétaire général des Nations Unies (2000), "Rapport du Millénaire ", en ligne : Organisation des Nations Unies <http://www.un. org/french/millenaire/sg/report/ full.htm>. 
cinquième indicateur que nous citerons concerne la pollution. En effet, "le quart des communautés ont découvert des traces de polluants dans leur eau à tests "13. En liaison avec la pollution, une source de risque importante, qui n'est pas abordé par l'enquête, est le réchauffement climatique. Son impact sur la santé a été discuté lors de la 7ème session annuelle de l'Instance permanente des Nations Unies sur les questions autochtones, au printemps 200814, ainsi que par le Conseil des droits de l'homme $^{15}$. En outre, l'espérance de vie des Autochtones est plus basse que celle des Québécois non-autochtones ${ }^{16}$.

\section{L'intensité du risque}

Chaque indicateur pris isolément illustre un aspect du risque en santé chez les peuples autochtones du Québec. Considérés ensemble, ces indicateurs dessinent un risque d'une toute autre étendue, en raison de l'accumulation des manifestations et de l'ampleur du phénomène. L'intensité du risque provient de la multiplicité et de l'accumulation des facteurs, mais aussi de leur enchevêtrement. Cette corrélation est mis en évidence par deux illustrations. D'une part, le caractère restreint

13. "Enquête régionale longitudinale sur la santé des Premières Nations de la région du Québec ", supra note 5 à la p. iii.

14. Le thème spécial de la 7 e session de l'Instance permanente sur les questions autochtones s'intitulait Les changements climatiques, diversité bioculturelle et moyens d'existence : le rôle de gardien des peuples autochtones et les nouveaux défis à relever. Pour plus d'informations sur les conclusions de cette rencontre, voir le site Internet de l'Instance: <http://www.un.org/esa/socdev/unpfii/fr/session_ seventh.html>. Voir également le rapport State of the World's Indigenous Peoples 2009, Secrétariat de l'Instance permanente des Nations Unies sur les questions autochtones, State of the World's Indigenous Peoples, ST/ESA/328, <http://www.un.org/esa/socdev/unpfii/documents/SOWIP_web.pdf>, United Nations, New York, 2009, aux pp. 95 et ss.

15. Doc. off. CDH, $7^{\mathrm{e}}$ sess., $21^{\mathrm{e}}$ séance, rec. A/HRC/7/L.21/Rev.1, (26 mars 2008).

16. Les membres des Premières nations du Québec et du Labrador vivent six à sept ans de moins en moyenne que le reste de la population du Québec, "Dévoilement d'un plan pour s'attaquer aux problèmes sociaux et de santé ", en ligne : C.S.S.S.P.N.Q.L. <http://www.cssspnql.com/ fr/nouvelles/plan2007-2017.htm>. 
Le triptyque santé

- identités autochtones -

105

territoire. Perspectives juridiques

du territoire est source de pauvreté et de marginalisation. Il en résulte un accroissement du nombre de personnes par logement et des conditions de vie insalubres dues à la pauvreté et à l'exclusion. À leur tour, ces deux tendances alimentent le risque en santé, en créant par exemple un terrain favorable au surpeuplement ainsi qu'à certaines maladies comme la tuberculose ${ }^{17}$. L'accès limité aux services de santé accentue encore

17. Le taux de tuberculose, entre 2002 et 2006 , est plus important chez les peuples autochtones du Canada que chez les canadiens nonautochtones. Il est, en effet, 29 fois plus élevé que chez les canadiens non-autochtones et, en ce qui concerne le peuple Inuit, 90 fois plus haut. "Bon nombre de facteurs favorisant la persistance de la tuberculose parmi les peuples autochtones du monde entier sont liés aux défis auxquels sont confrontées les Premières nations du Canada. Par exemple, un membre adulte des Premières nations sur cinq n'a pas accès à un médecin ni à une infirmière. De toute évidence, nous devons améliorer l'accès aux soins de santé, a déclaré Phil Fontaine, chef national [de l'Assemblée des Premières nations]. Cependant, pour être viables, les solutions doivent porter également sur les facteurs sociaux déterminants pour la santé, comme le logement et l'alimentation. En retour, les possibilités d'emplois et la richesse qu'ils engendrent sont des éléments clés pour créer des peuples sains et des communautés viables ". "Il est inacceptable que le taux de tuberculose au sein des communautés Inuits soit 90 fois plus élevé que pour l'ensemble des Canadiens. Le manque de logements adéquats et la surpopulation contribuent à faire grimper ce taux et la situation ne s'améliorera pas tant que nous n'aurons pas abordé ces problèmes ainsi que d'autres facteurs déterminants pour la santé. Notre passé en ce qui a trait à la tuberculose est tragique et, malheureusement, la situation est toujours la même aujourd'hui pour bon nombre de communautés Inuits ", a déclaré Mary Simon, chef nationale inuit ", Assemblée des Premières Nations, "Les chefs autochtones exigent des mesures pour réduire le nombre de cas de tuberculose et de décès liés à cette maladie " (13 novembre 2008), en ligne : <http://www.afn.ca/article.asp?id=4379>. Un rapport spécial du Comité canadien de lutte antituberculeuse informe également cette situation : Santé Canada, Rapport spécial du Comité canadien de lutte antituberculeuse, La tuberculose chez les peuples autochtones du Canada, en ligne: <http://www.phac-aspc.gc.ca/publicat/tbcbaptbpac/pdf/ SpecialReport-F.pdf>. La C.S.S.S.P.N.Q.L. indique également que "Les problèmes respiratoires, incluant l'asthme, les bronchites chroniques, l'emphysème et la tuberculose, sont parmi les maladies qui touchent une large population d'individus âgés entre 18 et 29 ans ", Enquête régionale longitudinale sur la santé des Premières Nations de la région du Québec, rapport des Premières nations vivants dans les 
ce risque. D'autre part, la pauvreté, la marginalisation et l'absence de reconnaissance par la société dominante portent atteinte à la possibilité de se projeter, de construire et de croire en un projet de vie. Ceci explique en partie l'existence d'un risque plus élevé d'échec, de mal-être conduisant à la dépression et au suicide ${ }^{18}$.

\section{Deux niveaux de risques et une source principale}

Au vue de ce qui précède, le risque en santé chez les Premières nations du Québec provient d'un climat défavorable lié à la perte d'identité. Il semble se situer à deux niveaux, d'abord, en termes d'obstacles à l'acquisition de conditions de santé suffisantes et égales à celles des allochtones ${ }^{19}$, ce qui fait référence aux négligences de l'État en matière d'intervention.

Ensuite, le risque en santé se situe à un second niveau. Il provient du fait que les services publics de santé, lorsqu'ils sont présents et accessibles, ne sont pas nécessairement adéquats. En effet, ils sont fondés sur les systèmes et les pratiques de santé ainsi que des valeurs allochtones, conduisant ainsi à négliger les pratiques et conceptions autochtones. Karine Gentelet, Guy Rocher et Alain Bissonnette ont mis en évidence la conception globale et holiste de la santé des Innus et des Atikamekws du Québec. Ils indiquent que :

communautés (2002), en ligne : C.S.S.S.P.N.Q.L <http://www.cssspnql. com/fr/recherche/ documents/ERS2002-Surcommunaute.pdf $>$ à la p. 119.

18. Carlos Coloma, Gilles Bibeau et Duncan Pedersen, La mortalité par suicide dans les communautés Atikamekw, Rapport présenté au Conseil de la Nation Atikamekw, Services sociaux, GIRAME et Centre de recherche de l'Hôpital Douglas, 1999, à propos du suicide au sein des communautés autochtones.

19. Les allochtones sont toutes les personnes qui ne sont pas autochtones. Ce terme est donc l'antonyme d'autochtone, selon le grand dictionnaire terminologique. Ce terme signifie littéralement terres d'ailleurs, alors que le terme autochtone fait référence aux personnes issues du sol même où elles habitent. Voir "Allochtone", en ligne: Le grand dictionnaire terminologique <http://www.granddictionnaire.com/btml/fra/r_motclef/ index1024_1.asp>. 
$\begin{array}{cc}\text { (2009-10) } 40 \text { R.D.U.S. } & \text { Le triptyque santé } \\ \text { - identités autochtones - } \\ \text { territoire. Perspectives juridiques }\end{array}$

[...] beaucoup d'interlocuteurs ont une conception active de la santé. Pour eux, la santé n'est avant tout ni l'absence de maladie, ni l'absence de malaise. Elle est plutôt la capacité d'agir, l'aptitude à fonctionner. Cette capacité d'agir s'exprime à trois niveaux de vie. Au plan individuel d'abord: être en mesure de vaquer à ses affaires, à ses diverses activités. Au niveau social ensuite : la santé s'exprime dans l'aptitude à interagir normalement avec toutes les autres personnes de son entourage. Et d'une manière plus négative, on est en santé quand on n'est pas un poids pour ceux avec qui l'on vit. Enfin, la santé consiste à être actif dans son environnement physique immédiat et plus éloigné, son territoire notamment, tout autant qu'avec son milieu social ${ }^{20}$.

La conception de la santé étant différente de celle proposée par les services publics, il en résulte une certaine inadéquation.

De plus, l'imposition de pratiques souvent uniformes nonautochtones participe au processus d'assimilation et est un frein à la reconnaissance des identités autochtones. Elle est source de risque en santé, parce qu'elle contraint, d'une part, les membres des communautés autochtones à user d'un service de santé qui n'est pas légitime à leurs yeux ${ }^{21}$, ce qui n'est pas propice à son utilisation. Ce n'est pas propice non plus à l'établissement d'un projet médical ou thérapeutique fondé sur la confiance. D’autre part, la consultation de ces services, lorsqu'elle a lieu, participe à l'assimilation des Autochtones et à l'abandon de leur savoir médical. Or, l'assimilation est elle-même source de risques, parce qu'elle est une forme de non-reconnaissance identitaire, dommageable émotionnellement, socialement et psychologiquement. Elle est également dommageable sur le plan collectif, puisqu'elle conduit à l'amenuisement de l'identité. Cet aspect du risque en santé questionne d'une autre façon l'intervention de l'État. À la différence du premier, qui porte sur les négligences des autorités, il conduit à s'interroger sur la pertinence et la légitimité

20. Gentelet, Bissonnette et Rocher, supra note 2 aux pp. 95 et 96.

21. Ibid. aux pp. 100-104, sur la différence entre "biomédecine " et " médecine traditionnelle " et sur leur différence en termes de légitimité. 
de cette intervention : comment organiser, proposer et instaurer un service de santé adéquat et légitime en territoire autochtone?

Les deux niveaux de risque ont une source commune, qui est la colonisation et ses effets contemporains ${ }^{22}$, conduisant à l'exclusion, la dépossession des territoires, de la culture et du savoir, la marginalisation, la pauvreté et la non-reconnaissance. Il est avéré que la colonisation est un phénomène destructeur sur le plan individuel et collectif 23 , par la subordination, l'anéantissement identitaire et les dommages psychologiques et sociaux qu'elle engendre. La colonisation constitue donc la source initiale de risque en santé et donne elle-même naissance à une multitude de "sous-sources". Elle est entretenue par l'ensemble des institutions sociales dont le droit.

22. La colonisation est le fait d'imposer de l'extérieur, de façon impérialiste une vision du monde, négligeant les points de vue autochtones. Elle implique la nécessité de coexister avec d'autres venus d'ailleurs, une réalité contraignante. Elle entraine l'usurpation des territoires, la restriction des espaces, la marginalisation économique, sociale, culturelle, spirituelle et identitaire ainsi que la pauvreté, qui sont à leur tour des facteurs amplifiant le risque en santé. La colonisation est un phénomène sous-jacent et tabou, mais qui est toujours à l'œuvre, parce qu'il n'est pas abordé ni reconnu. Elle implique un décalage entre les dynamiques sociales majoritaires et autochtones. Dans ces circonstances, le dialogue et la réconciliation effective ne sont pas possibles. À ce titre, certains auteurs indiquent que "la vie sur la réserve, en communauté fermée, qui leur a été imposée par la colonisation, est perçue comme particulièrement pathogène pour les adultes et sans doute encore pour les jeunes. La violence, les suicides, l'alcoolisme et le recours aux drogues lui sont particulièrement attribuables ", Gentelet, Bissonnette et Rocher, supra note 2 à la p. 98.

23. James Sa'ke'j Henderson Youngblood, et Wanda D. McCaslin, "Exploring Justice as Healing ", dans Wanda D. McCaslin, dir., Justice as Healing. Indigenous Ways, St Paul, Living Justice Press, 2005 aux pp. 4-7 [Wanda D. McCaslin]; Charles Taylor, Multiculturalisme, différence et démocratie, Champs, Flammarion, 1992. 
Le triptyque santé

(2009-10) 40 R.D.U.S. - identités autochtones -

109

territoire. Perspectives juridiques

\section{Globalité du risque}

Par conséquent, nous adhérons à une vision étendue du concept de risque en santé24. En tant que phénomène non-isolé, il doit être pensé dans un contexte de risque généralisé généré par la colonisation, l'exclusion sociale et la non-reconnaissance ${ }^{25}$. Il se manifeste à travers les problèmes de santé, en termes de maladies, de carences ou de déficiences, mais aussi à travers l'existence de troubles psychologiques et sociaux. À ce titre, la conclusion du rapport de la C.S.S.S.P.N.Q.L. est claire :

De nombreuses communautés sont encore aujourd'hui confrontées à des problèmes criants qui minent leur accès à des besoins fondamentaux et à des conditions parfois comparables à celles du tiers monde.

Pour toutes ces raisons, les membres des communautés des Premières nations du Québec bénéficient d'une qualité de vie réduite à plusieurs égards. Aussi, un bon nombre des problèmes de santé et des problèmes psychosociaux présents dans certaines communautés peuvent être attribuées en partie à un milieu de vie déficient. [...]

24. Nous référons au risque en santé ou au risque sanitaire de manière indissociable, bien que la seconde appellation soit plus étendue. Cependant, le risque en santé est un risque sanitaire.

25. "Many illnesses that have a disproportionate impact on indigenous peoples, especially problems of mental health, are related to the colonialist and racist structures that cause indigenous communities to be some of the poorest and most marginalized in the world. Not only have indigenous peoples experienced a collective history of genocide, dispossession and dislocation, manifestations of these violent forces persist today in the form of development aggression, forced displacement and economic exploitation ", Secrétariat de l'Instance permanente des Nations Unies sur les questions autochtones, State of the World's Indigenous Peoples, ST/ESA/328, New York (2009) en ligne: United Nations <http://www.un.org/esa/socdev/ unpfii/documents/SOWIP_ web.pdf> à la p. 167. 
Tous les problèmes mentionnés [...], et bien d'autres, contribuent grandement à l'effritement de la qualité de vie dans les communautés ${ }^{26}$.

Cela implique que le risque soit conçu de manière globale.

Nous venons de circonscrire le risque sanitaire en contexte autochtone. Expliquons maintenant de quelle manière le droit intervient dans sa reproduction.

\section{Le droit et le risque en santé chez les peuples autochtones}

Le droit est un espace qui reflète les valeurs et les tendances sociopolitiques dominantes ${ }^{27}$. Il peut alors entretenir le

26. Enquête régionale longitudinale sur la santé des Premières Nations de la région du Québec, rapport sur l'influence du milieu sur la santé des Premières nations (2002), en ligne: C.S.S.S.P.N.Q.L. $<$ http ://www.cssspnql.com/fr/recherche/documents/ERS2002-Influenc edumilieu.pdf>, supra note 5 à la p. 39. Certains chercheurs ont adopté des positions similaires. Par exemple, James Tully ou Charles Taylor. James Tully explique, par exemple, à propos des Autochtones que " [...] l'avilissement de leurs identités et le manque de respect à leur égard, sous les modalités du sexisme, du racisme, du nationalisme, du chauvinisme linguistique et culturaliste, du classement pseudoscientifique des cultures et des langues en fonction de leur niveau de développement, avec l'Europe et les États-Unis au sommet de la hiérarchie, ainsi que le fait d'imposer des cultures dominantes par des processus détruisant les identités, assimilant ou marginalisant des individus et des groupes, tout cela n'est pas seulement injuste. Cela sape également le respect de soi et, par conséquent, les capacités mêmes des individus concernés à résister à ces injustices, voire même à agir efficacement dans les perspectives d'une recherche d'assimilation. Ce sont là les causes des pathologies bien connues de l'oppression, de l'assimilation et de la marginalisation: manque d'estime et de respect pour soi-même, aliénation, pauvreté transgénérationnelle, consommation de drogue, chômage, destruction des communautés, taux élevés de suicide, etc. ", James, Tully, "Une étude de la politique de l'identité ", dans Will Kymlicka et Sylvie Mesure, dir., Les identités culturelles, Paris, PUF, 2000 à la p. 199.

27. En ce sens, Andrée Lajoie, Jugements de valeurs, Paris, P.U.F., 2007. 
$\begin{array}{cc}\text { (2009-10) } 40 \text { R.D.U.S. } & \text { Le triptyque santé } \\ \text { - identités autochtones - } \\ \text { territoire. Perspectives juridiques }\end{array}$

risque en santé, en étant inadapté à la diversité des situations et en intervenant en décalage par rapport au contexte autochtone.

Néanmoins, si nous observons la dynamique actuelle,
extérieure au droit écrit, nous constatons qu'une reconceptualisation de la relation entre l'État et les Autochtones, et ce, notamment, à travers l'établissement d'un dialogue mutuel et d'une collaboration, est en cours au Canada. Elle a pour effet de reconsidérer et de rééquilibrer le rôle que chacun peut jouer en matière de prise de décisions. Dans cet esprit, l'État considère davantage les Autochtones en tant que citoyens actifs - c'est-àdire aptes à apporter des perspectives valables pour la société toute entière. Cette approche est actuellement développée au Québec par l'établissement d'accords, qui permettent le développement d'initiatives autochtones. Il s'agit, par exemple, des accords de contribution proposés par le gouvernement fédéral ou des ententes de principes conclues entre les paliers fédéral et provincial ainsi que les communautés autochtones ${ }^{28}$.

28. Plusieurs exemples peuvent être cités au Québec. Voir notamment l'entente intermédiaire conclues entre le Conseil (tribal) de la Nation Atikamekw - Atikamekw sipi, le Centre jeunesse Mauricie et Centre-duQuébec, le Centre jeunesse Lanaudière et leurs directions de la protection de la jeunesse, entente qui a permis le développement du Système d'Intervention d'Autorité Attikamekw (SIAA), Andrée Lajoie, Le rôle des femmes et des aînés dans la gouvernance autochtone au Québec, Montréal, Thémis, 2009 aux pp. 51-53.

Sur les expériences en matière de coopération et sur la complémentarité des représentations et des pratiques, Gentelet, Bissonnette et Rocher, supra note 2 aux pp. 106-110. Les auteurs indiquent notamment que "Cette dichotomie des identités et des valeurs n'a pourtant pas empêché l'émergence de nouvelles pratiques. Au contraire, elle a favorisé de nouveaux fonctionnements au sein même des institutions de santé des quatre communautés. En effet, la reconnaissance de la médecine traditionnelle au sein du personnel médical a permis la mise en place de mesures visant l'harmonisation de la pratique de la biomédecine à celle de la médecine traditionnelle. Cette coopération entre les deux systèmes médicaux permet alors aux patients d'avoir accès à des soins qui partent de leurs besoins spécifiques et qui interviennent sur les plans social, biologique, émotionnel, spirituel et écologique ", supra note 2 à la p. 107. 
Toutefois, cette dynamique n'a pas toujours de répercussion sur les normes juridiques positives élaborées en droit interne. Par exemple, elle ne tend pas à renforcer juridiquement la maîtrise des territoires ancestraux par les Autochtones eux-mêmes, en créant un accès sécurisé par la reconnaissance d'un droit commun et uniforme au territoire. Ainsi, le risque en santé se reproduit, en raison, d'une part, de l'inexistence d'un droit d'accès " sécurisé " aux territoires ancestraux pour toutes les communautés et, d'autre part, du fait de décalages dans l'élaboration et la mise en œuvre du droit.

\section{Non reconnaissance en droit interne d'un accès sécurisé aux territoires ancestraux}

L'un des facteurs de risque en santé chez les populations autochtones se caractérise par l'inexistence d'un droit d'accès sécurisé aux territoires ancestraux. Cela provient de la mauvaise conception et de la non-application, en droit canadien, du principe de liberté des peuples à disposer d'eux-mêmes à l'égard des Autochtones $^{29}$. De ce fait, le point de vue des Autochtones est

29. Le principe d'autodétermination est, selon Jean Salmon, un " principe de nature politique d'inspiration démocratique désignant la vocation des peuples à s'administrer librement ". Il dispose, en droit international, de deux dimensions qui résultent en deux mises en œuvre différentes. Dans sa dimension interne, l'autodétermination permet à tous les peuples de s'administrer librement à l'intérieur même des frontières déjà établies. Ce premier aspect favorise le transfert de compétence vers des paliers de gouvernements locaux, les formes de décentralisation et la création de statuts d'autonomie. Cette dimension ne porte donc pas atteinte au principe d'intégrité des frontières nationales. A contrario, dans sa dimension externe, le principe d'autodétermination autorise un peuple à exercer son droit de session, c'est-à-dire à se séparer de l'État sous la domination duquel il vit pour former son propre État. Cette seconde dimension a initialement été exercée pour justifier la décolonisation des États d'Afrique. Dans le cas des peuples autochtones, bien que la Déclaration des Nations Unies sur les droits des peuples autochtones (articles 3 et 46) ainsi que la doctrine reconnaissent le principe d'autodétermination dans son aspect interne, certains États comme le Canada, craignent qu'il soit mis en ouvre, à leur égard, dans son aspect externe. Sur ce principe, lire Théodore Christakis, Le droit à l'autodétermination en dehors des situations de décolonisation, Monde 
$\begin{array}{cc}\text { (2009-10) } 40 \text { R.D.U.S. } & \text { Le triptyque santé } \\ \text { - identités autochtones - } \\ \text { territoire. Perspectives juridiques }\end{array}$

souvent imperceptible, méconnu et dépassé par des intérêts plus puissants, tels que les intérêts économiques du pays. Une application réaliste du principe de liberté des peuples à disposer d'eux-mêmes va notamment dans le sens d'une écoute et d'une association plus importante des Autochtones à la prise de décisions et à la gestion des services ${ }^{30}$. Le Canada et la province du Québec développent cette approche. Rappelons qu'ils ont signé avec diverses communautés autochtones de la province des ententes de principe et qu'un devoir de consultation a été imposé aux autorités par la Cour suprême ${ }^{31}$. Néanmoins, les ententes représentent un règlement casuistique, à l'instar d'une reconnaissance de l'autodétermination, entendue en tant que principe permettant une coexistence plus équitable et plus respectueuse de chacune des entités composant la société. Quant au devoir de consultation, celui-ci dispose encore d'un caractère plus théorique que pratique, dans la mesure où les consultations

européen et international, C.E.R.I.C., 1999; Antonio Cassese, Selfdetermination of peoples: A legal Reappraisal, Cambridge, Cambridge University Press, 1995; Jean Salmon, Dictionnaire de droit international public, Bruxelles, Bruylant, 2001. Voir également la différence pertinente que fait Alexandra Xanthaki, Indigenous Rights and United Nations Standards. Self- Determination, Culture and Land, Cambridge, Cambridge University Press, 2007 aux pp. 155-157 et James Anaya, Indigenous Peoples in International Law, Oxford, Oxford University Press, 2004 aux pp. 98-105.

30. Le Secrétariat de l'Instance permanente des Nations Unies sur les questions autochtone recommande : "All these experiences tell us that intercultural health systems must be based on building the autonomy and ensuring the empowerment of indigenous peoples, which derive from the full recognition and exercise of rights of indigenous peoples. The framework for an intercultural system therefore includes selfdetermination; sovereignty over land, territory and natural resources; full and effective participation in decision-making arenas (including processes based on the principle of free, prior and informed consent); the recognition of indigenous health definitions and norms; and the recognition and protection of collective traditional knowledge ", Secrétariat de l'Instance permanente des Nations Unies sur les questions autochtones, State of the World's Indigenous Peoples, ST/ESA/328 (2009), en ligne: United Nations <http://www.un.org/esa/ socdev/unpfii/documents/SOWIP_ web.pdf> aux pp. 179 et 180.

31. Nation Haïda c. Colombie-Britannique (Ministre des Forêts), [2004] 3 R.C.S. 511. 
de communautés autochtones, qui se déroulent antérieurement à l'autorisation d'exploiter un territoire, pérennisent souvent le rapport de force existant entre ces communautés et l'État. Dans un article récent sur les négociations entres l'État et les communautés autochtones en matière de gouvernance, Michael Coyle souligne :

[...] un certain nombre d'obstacles réduisent les probabilités que les négociations sur la gouvernance génèrent des résultats reflétant véritablement les besoins et les aspirations des autochtones. Le plus important d'entre eux provient sans doute du déséquilibre entre les capacités des parties à promouvoir leurs intérêts en dehors de la table de négociation, un élément qui donne à l'État un pouvoir de négociation disproportionné vis-à-vis des résultats espérés en matière de gouvernance. En outre, puisqu'il n'est pas assuré que les parties percevront leurs objectifs comme complémentaires, le contexte de rapports de force accroît le risque que les négociations ne débouchent pas sur une entente, ou que les ententes obtenues ne fassent guère avancer les intérêts des deux parties. Finalement, les défis systémiques, culturels et cognitifs tendent à diminuer plus encore l'effet des aspirations autochtones sur les résultats des négociations sur la gouvernance.

Les défis soulevés par les rapports de force entre les parties et par leurs relations culturelles sont très souvent exacerbés par leur histoire coloniale. Cette histoire, nourrie de la domination politique et juridique de l'État, laisse entendre que les négociations sur la gouvernance se dérouleront fréquemment dans une atmosphère de méfiance. Le manque de confiance entre les participants concourt à les prédisposer à l'emploi de tactiques défensives et agressives et à affaiblir d'autant l'efficacité des initiatives visant au partage de l'information, à une écoute attentive et à la résolution en commun des problèmes. Ces multiples écueils compromettent les négociations sur la gouvernance, dont le dessein, après tout, est la reconnaissance et la protection de la singularité identitaire autochtone, la création de modèles 
$\begin{array}{cc}\text { (2009-10) } 40 \text { R.D.U.S. } & \text { Le triptyque santé } \\ \text { - identités autochtones - } \\ \text { territoire. Perspectives juridiques }\end{array}$

inusités de gouvernance et le renouveau de la relation entre l'État et les peuples autochtones ${ }^{32}$.

Par conséquent, autodétermination et diminution du risque en santé ont une relation, dans la mesure où la mise en œuvre de ce principe implique à la fois l'instauration d'une relation de confiance entre l'État et les Autochtones, l'aboutissement du processus de reconnaissance et la consécration d'un accès sécurisé au territoire, trois éléments propres à établir un climat favorable au bien-être et à la diminution du risque ${ }^{33}$. Par ailleurs, l'entretient du risque en santé ne se produit pas seulement par la non reconnaissance en droit interne d'un accès sécurisé aux territoires ancestraux, via le principe d'autodétermination. Il se produit également par d'autres mécanismes, touchant à l'élaboration et à la mise en ouvre du droit.

32. Michael Coyle, "Les négociations sur la gouvernance autochtone au Canada: pouvoir, culture et imagination" (2009) Téléscope 15(3), en ligne : $\quad$ ENAP < http://www.enap.ca/OBSERVATOIRE/fr/telescopemenus. aspx?sortcode=1.16.18.19> à la p. 25.

33. À ce propos, le rapport du Secrétariat de l'Instance permanente des Nations Unies sur les questions autochtones, State of the World's Indigenous Peoples 2009 indique, en effet, concernant le Canada que "Canada recognizes that key socio-economic indicators for Aboriginal people are unacceptably lower than for non-Aboriginal Canadians. [...] The restrictions put on Aboriginal peoples' ability to protect, meaningfully benefit from and freely dispose of their land and resources constitute the main obstacle to real economic development among First Nations, Métis and Inuit. As a result of land loss and severe limitations set by the various levels of government on the free use of and continuing benefit from their natural resources, Aboriginal people have become increasingly dependent on welfare measures undertaken by the federal or provincial governments. This accounts for the large disparities between Aboriginal people and other Canadians ", Secrétariat de l'Instance permanente des Nations Unies sur les questions autochtones, State of the World's Indigenous Peoples, ST/ESA/328, New York (2009), en ligne : United Nations <http://www.un.org/esa/socdev/ unpfii/documents/SOWIP web.pdf> aux pp. 24 et 25. 


\section{Entretient du risque en santé par d'autres mécanismes juridiques}

L'élaboration et la mise en œuvre du droit ont des répercussions sur le risque en santé chez les peuples autochtones du Québec. Ceci nous confronte à la nécessité de transformer le droit afin de le rendre plus inclusif 34 . La législation canadienne pérennise le risque en santé chez les populations autochtones et ce, à trois niveaux, d'abord, en niant ou en négligeant les identités autochtones, ensuite, par son manque de perspective holiste, enfin, par la faiblesse des droits économiques et sociaux en droit interne.

Premièrement, le législateur peut contribuer à la formation ou à l'entretien du risque en santé, en niant ou en négligeant les identités autochtones. D'abord, le droit interne canadien organise le processus d'assimilation et la fragmentation des communautés, à travers la Loi sur les Indiens ${ }^{35}$, dans la mesure où il encadre l'attribution ou la non-attribution du statut d'Indien ${ }^{36}$. Comme le rapport sur l'influence du milieu sur la santé des Premières nations le souligne, l'article 6 de cette loi retire le statut d'Indien à tout Autochtone qui serait le produit de deux générations de métissage $^{37}$. En d'autres termes, si un enfant $Z$ provient d'un

34. Nous n'avons pas la prétention ici de proposer un modèle de transformation, mais seulement de cerner ce qui ne fonctionne pas.

35. Loi sur les Indiens, L.R.C. 1985 c. I-5. [Loi sur les Indiens]

36. Malgré son caractère inadéquat et critiquable, nous utilisons le terme " statut d'Indien " parce que c'est celui qui est consacré par la Loi sur les Indiens.

37. Loi sur les Indiens, art. 6 : " (1) Sous réserve de l'article 7, une personne a le droit d'être inscrite si elle remplit une des conditions suivantes :

a) elle était inscrite ou avait le droit de l'être le 16 avril 1985;

b) elle est membre d'un groupe de personnes déclaré par le gouverneur en conseil après le 16 avril 1985 être une bande pour l'application de la présente loi;

c) son nom a été omis ou retranché du registre des Indiens ou, avant le 4 septembre 1951, d'une liste de bande, en vertu du sous-alinéa 12(1)a)(iv), de l'alinéa $12(1) \mathrm{b}$ ) ou du paragraphe 12(2) ou en vertu du sous-alinéa 12(1)a)(iii) conformément à une ordonnance prise en vertu du paragraphe 109(2), dans leur version antérieure au 17 avril 1985, ou en vertu de 
$\begin{array}{cc}\text { (2009-10) } 40 \text { R.D.U.S. } & \text { Le triptyque santé } \\ \text { - identités autochtones - } \\ \text { territoire. Perspectives juridiques }\end{array}$

parent Autochtone détenant le statut d'Indien (Y) et d'un parent non-autochtone $(\mathrm{X})$ et que le parent autochtone(Y) est lui-même issu d'un couple métissé (c'est-à-dire dans lequel un seul des parents détenait le statut), l'enfant $Z$ perdra le statut d'Indien définitivement et ne pourra le transmettre. Il ne pourra pas non plus résider sur la réserve, lieu de contact avec le territoire ancestral et les membres de sa communauté. Or, la fragmentation des communautés porte atteinte à la transmission de l'identité, et la loi empêche la reconnaissance officielle de celle-ci à l'égard de certaines personnes qui se considèrent pourtant comme Autochtones et qui sont reconnues en tant que tel par leur communauté d'appartenance. Cette loi encadre la détermination juridique de l'identité autochtone et dépossède en cela les

toute disposition antérieure de la présente loi portant sur le même sujet que celui d'une de ces dispositions;

d) son nom a été omis ou retranché du registre des Indiens ou, avant le 4 septembre 1951, d'une liste de bande, en vertu du sous-alinéa 12(1)a)(iii) conformément à une ordonnance prise en vertu du paragraphe 109(1), dans leur version antérieure au 17 avril 1985, ou en vertu de toute disposition antérieure de la présente loi portant sur le même sujet que celui d'une de ces dispositions;

e) son nom a été omis ou retranché du registre des Indiens ou, avant le 4 septembre 1951, d'une liste de bande :

(i) soit en vertu de l'article 13, dans sa version antérieure au 4 septembre 1951, ou en vertu de toute disposition antérieure de la présente loi portant sur le même sujet que celui de cet article,

(ii) soit en vertu de l'article 111 , dans sa version antérieure au ler juillet 1920, ou en vertu de toute disposition antérieure de la présente loi portant sur le même sujet que celui de cet article;

f) ses parents ont tous deux le droit d'être inscrits en vertu du présent article ou, s'ils sont décédés, avaient ce droit à la date de leur décès.

(2) Sous réserve de l'article 7, une personne a le droit d'être inscrite si l'un de ses parents a le droit d'être inscrit en vertu du paragraphe (1) ou, s'il est décédé, avait ce droit à la date de son décès.

(3) Pour l'application de l'alinéa (1)f) et du paragraphe (2) :

a) la personne qui est décédée avant le 17 avril 1985 mais qui avait le droit d'être inscrite à la date de son décès est réputée avoir le droit d'être inscrite en vertu de l'alinéa (1)a);

b) la personne visée aux alinéas (1)c), d), e) ou f) ou au paragraphe (2) et qui est décédée avant le 17 avril 1985 est réputée avoir le droit d'être inscrite en vertu de ces dispositions ". 
communautés ainsi que leurs membres de ce rôle38. À ce titre, la Loi sur les Indiens est le produit d'une vision homogène et décalée de l'identité.

De manière similaire, les dispositions relatives aux ordonnances de placement permanent contenues dans la Loi sur la protection de la jeunesse (projet de loi 125) ont un effet similaire ${ }^{39}$. Cette loi entraine le placement d'enfants autochtones dans des familles non-autochtones. Comme l'article 6 de la Loi sur les Indiens, elle a pour effet de diviser les communautés et de séparer leurs membres. Le placement d'enfants autochtones dans des familles non-autochtones provoque un déracinement et porte atteinte à la transmission de l'identité d'origine. L'enfant peut disposer $\mathrm{du}$ statut d'Indien, mais se trouve déconnecté de son identité. De plus, la Loi sur la protection de la jeunesse entérine une situation de discrimination, dans la mesure où les enfants autochtones sont davantage concernés par le placement en famille d'accueil. Or, cette situation est souvent due aux conditions de pauvreté dans lesquelles vivent les communautés autochtones, résultant des négligences de l'État ${ }^{40}$. Conséquemment, tant

38. On peut s'interroger sur la légitimité de l'État à déterminer qui est autochtone et qui ne l'est pas.

39. Assemblée des Premières Nations du Québec et du Labrador, Communiqué, "Traitement inéquitable réservé aux familles et aux enfants des Premières Nations - Les Premières Nations demandent une enquête à la Commission des droits de la personne et de la jeunesse " (20 juin 2008) [Traitement inéquitable].

40. À ce titre, le communiqué de l'Assemblée des Premières Nations du Québec et du Labrador cite quelques chiffres révélateurs :

" Au cours des cinq dernières années, sur une population de 12094 enfants, 1404 enfants des Premières Nations sont annuellement placés.

- De ces 1404 enfants placés annuellement, 429 d'entre eux ont cinq ans et moins.

- Ils auront passé 189036 jours en ressource de placement, pour une durée moyenne de placement de 9,5 mois par enfant.

- Au Québec, en 2002-2003, les enfants des Premières Nations étaient 7 fois plus placés à l'extérieur de leur famille que les enfants québécois.

- On estime qu'en 2008-2009, ils seront neuf fois plus sujets à des placements et l'écart tend à augmenter de 0,33 fois par année.

- Les causes les plus fréquentes de placement sont directement liées à la pauvreté et au manque de ressources locales. C'est aussi pour les mêmes 
$\begin{array}{cc}\text { (2009-10) } 40 \text { R.D.U.S. } & \text { Le triptyque santé } \\ \text { - identités autochtones - } \\ \text { territoire. Perspectives juridiques }\end{array}$

l'article 6(3) de la Loi sur les Indiens que la Loi sur la protection de la jeunesse contribuent à la perte ou à l'amenuisement de l'identité autochtone, ce qui représente une source de risque psychosocial.

En outre, le partage des compétences tel qu'il est organisé par les articles 91 et 92 de la Loi constitutionnelle du Canada de 1867, crée un risque en santé, dans la mesure où la compétence sur les questions autochtones appartient au palier fédéral de gouvernement ${ }^{41}$ alors que la compétence en santé est attribuée au palier provincial ${ }^{42}$. Les provinces réglementent la santé et gèrent les services en la matière. Or, lorsqu'un problème intervient dans ce domaine et qu'il implique un membre d'une communauté autochtone, le palier fédéral s'en remet parfois à la compétence provinciale en santé et n'intervient pas lui-même et vice versa ${ }^{43}$. La répartition des compétences est source de discrimination et de disfonctionnements. En effet, les ressortissants autochtones ne peuvent bénéficier des mêmes droits d'accès aux services de santé, tels qu'ils sont organisés par la province, puisqu'en raison de leur identité, elle n'est pas compétente et ne les considère pas comme étant bénéficiaire de ses services. À travers ces exemples, on

raisons que la plupart des placements sont effectués à l'extérieur du milieu culturel et social de l'enfant ", voir Traitement inéquitable, supra note 39.

41. Loi constitutionnelle de 1867 (R.-U.), 30 \& 31 Vict., c. 3, art. 91(24), reproduit dans L.R.C. 1985, app. II, no 5 : "[...] il est par la présente déclaré que (nonobstant toute disposition contraire énoncée dans la présente loi) l'autorité législative exclusive du Parlement du Canada s'étend à toutes les matières tombant dans les catégories de sujets cidessous énumérés, savoir [...] Les Indiens et les terres réservées pour les Indiens".

42. Ibid., art. 92 (7) : "Dans chaque province la législature pourra exclusivement faire des lois relatives aux matières tombant dans les catégories de sujets ci-dessous énumérés, savoir : [...] l'établissement, l'entretient et l'administration des hôpitaux, asiles, institutions et hospices de charité dans la province, autres que les hôpitaux de marine".

43. Constance MacIntosh, "Jurisdictional Roulette: Constitutional and Structural Barriers to Aboriginal Access to Health ", dans Colleen Flood (dir.), Just Medicare, Toronto, University of Toronto Press, 2006 aux pp. 196-198. 
constate ainsi que le droit interne et son ordonnancement ont des lacunes qui sont autant de facteurs d'assimilation, de fragmentation et de discrimination.

Deuxièmement, la reproduction du risque sanitaire est accentuée par le manque de perspective juridique holiste aboutie quant aux identités autochtones ${ }^{44}$. En d'autres mots, dans la perspective autochtone les droits aux territoires, aux ressources naturelles, à des modes de vie propres, à la spiritualité et à une identité sont interconnectés. Or, les systèmes juridiques internes ou supranationaux ne reconnaissent pas nécessairement l'ensemble de ces droits et les considèrent souvent de manière indépendante les uns des autres. Rappelons par exemple que l'ordre juridique canadien ne reconnaît pas de droit au territoire à chacune des communautés autochtones. Il néglige, en ce sens, l'importance de leur relation au territoire, pourtant, au fondement de la reconnaissance identitaire et du bien être des Autochtones. De fait, le droit a tendance à cloisonner les normes et à favoriser les intérêts majoritaires. Il reproduit en cela le risque en santé, dans la mesure où santé, reconnaissance identitaire et territoire sont interconnectés.

Cette relation a néanmoins été mise en exergue par certaines décisions de la Cour interaméricaine des droits de l'homme, par exemple, dans une affaire qui impliquait une communauté Maroons et le Suriname ${ }^{45}$. La décision Moiwana village $v$. Suriname est pertinente, car elle reconnaît cette relation de manière très claire ${ }^{46}$. En 1986, l'attaque par l'armée du

44. Bien qu'il faille nuancer cette affirmation concernant le droit international, puisque diverses organisations supranationales, notamment les Nations Unies et ses institutions spécialisées, discutent par exemple des effets des changements climatiques sur la santé des individus et des peuples autochtones. Voir supra notes 14 et 15.

45. Moiwana Village $v$. Suriname, requête no 11821 , arrêt, (15 juin 2005), en ligne: Cour interaméricaine des droits de l'homme [C.I.D.H.] <http://www.corteidh.or.cr/docs/casos/articulos/seriec_124_ing.pdf> [Suriname].

46. Néanmoins, le Comité des droits de l'homme des Nations Unies [C.D.H.] avait également reconnu l'impact de l'exploitation du territoire sur le 
Le triptyque santé

- identités autochtones -

territoire. Perspectives juridiques

Suriname du village Maroons de Moiwana cause la mort violente d'une grande partie de la population. Les corps des défunts sont brûlés. Les survivants fuient le village, en raison de craintes liées à une attaque ultérieure et des menaces de l'armée ${ }^{47}$. Or, pour le peuple Maroons comme pour de nombreux autres, la conservation, par la communauté, des corps des défunts est fondamentale. L'accomplissement de cérémonies l'est aussi. Elles permettent la célébration des défunts, empêchent la rupture des liens entre vivants et morts, issus d'une conception du rapport au temps qui ne s'arrête pas à la vie, et démontre l'honneur que la communauté porte à ses défunts. En outre, la crémation est une offense. Dans le cas de la communauté Maroons de Moiwana, ces

mode de vie, les conditions de vie et la santé des membres du peuple Lubicon, mais de façon plus timide toutefois. Voir à ce titre, Ominayak and the Lubicon Lake Band $v$. Canada, (10 mai 1988), no 167/1984, en ligne: C.D.H. communication <http://www.unhchr.ch/tbs/doc.nsf/ (Symbol)/750d146488937439c1256ac50051a3d3?Opendocument>.

47. Les requérants appartiennent à la communauté Maroons N'djuka du Suriname. Celle-ci est originaire d'Afrique et fut conduite de force au Suriname lors de la colonisation européenne, au XVIIème siècle. Malgré cela, la relation qu'entretient la communauté avec la terre où elle demeure aujourd'hui est d'une importance culturelle, spirituelle et matérielle vitale (c'est pourquoi cet exemple nous semble appropriée et peut juridiquement servir de base à une comparaison avec les peuples autochtones). Les N'djuka fondent Moiwana au XIXème siècle. En 1986, dix camps composent le village. Ses habitants vivent de la chasse, de la pêche et de l'agriculture traditionnelle. Il s'agit d'une société matriarcale. En 1980, un coup d'État se produit au Suriname et un régime militaire est établi. Entre 1986 et 1987 de nombreux civils sont tués, principalement des membres du peuple Maroons. En 1986, nombre d'entre eux rejoignent un commando : "The Jungle Commando ". Pour répondre à celui-ci, l'armée va conduire de nombreuses attaques dans la région de Moiwana. C'est dans ces circonstances que le village N'djuka est attaqué le 29 novembre 1986. L'armée et les agents du gouvernement tuent 39 civils, notamment des femmes, des enfants et des aînés. Ils en blessent également beaucoup d'autres. Ils brûlent ensuite le village, détruisent les habitations et contraignent les survivants à fuir leurs terres. Certains seront déplacés à l'intérieur même du pays et d'autres rejoindront les camps de réfugiés en Guyane française. Depuis, ils vivent dans la pauvreté et n'ont plus de moyens de subsistance. En outre, le village est à l'abandon et ils n'ont pu retrouver les dépouilles des membres de la communauté tués lors de l'attaque. 
rituels n'ont pas pu être réalisés. Leur non-réalisation est ainsi vécue comme une transgression morale de la tradition et hante l'esprit des morts et des ancêtres, qui ne peuvent reposer en paix. Elle est également la cause de maladies "spirituelles " affectant les personnes qui ont survécu au massacre. Elles ont des conséquences sur la santé physique des descendants et des membres du lignage du défunt non inhumé.

Les faits de cette affaire marquent l'importance du rapport au territoire, au temps et aux autres et leur particularité. L'accès à la terre garantit l'intégrité et l'identité du peuple Maroons. La Cour reconnaît cette relation en condamnant l'État sur le fondement de l'article 5 de la Convention américaine des droits de l'homme4, portant sur le droit à des traitements humains, mais aussi sur celui de l'article 21 de la Convention ${ }^{49}$, sur le droit de propriété. La Cour impose également une obligation de délimiter et de démarquer le territoire, afin que la communauté puisse y exercer son droit de propriété collective. Elle reconnaît implicitement les conséquences en santé dues à l'absence d'accès au territoire et à la violation des coutumes, puisque celles-ci sont sources de

48. Convention américaine des droits de l'homme, art. : "1. Every person has the right to have his physical, mental, and moral integrity respected. 2 . No one shall be subjected to torture or to cruel, inhuman, or degrading punishment or treatment. All persons deprived of their liberty shall be treated with respect for the inherent dignity of the human person. [...] ".

49. Ibid., art. 21: "1. Everyone has the right to the use and enjoyment of his property. The law may subordinate such use and enjoyment to the interest of society. 2. No one shall be deprived of his property except upon payment of just compensation, for reasons of public utility or social interest, and in the cases and according to the forms established by law. 3. Usury and any other form of exploitation of man by man shall be prohibited by law". Depuis la décision Mayagna (sumo) Awas Tingni community v. Nicaragua, la Cour interaméricaine fait une interprétation extensive de cet article, qui lui permet de reconnaître le droit de propriété collective des territoires ancestraux, sur ce fondement, The Mayagna (sumo) Awas Tingni community v. Nicaragua, requête no 11577, arrêt, (31 août 2001), en ligne : C.I.D.H. <http://www.corteidh.or.cr/docs/casos/ articulos/seriec_79_ing.pdf $>$ [Nicaragua]. Cette interprétation est confirmée par la décision Moiwana village $v$. Suriname, du 15 juin 2005aux par. 131-135. 
Le triptyque santé

(2009-10) 40 R.D.U.S. - identités autochtones -

maladies dites "spirituelles" 50. La violation de l'identité, des conceptions profondes et des croyances d'un peuple, au fondement même de la socialisation de ses membres, a des conséquences en termes de souffrances émotionnelles, psychologiques et spirituelles, qui signalent toute l'étendue du risque en santé, dans ce cas d'espèce. La Cour semble y attribuer une grande importance en donnant gain de cause aux requérants. Toutefois, l'importance de cette relation et les enjeux qu'elle implique ne sont que rarement reconnus juridiquement.

Troisièmement, la situation actuelle des Premières nations du Québec, telle que décrite par l'enquête régionale longitudinale de la C.S.S.S.P.N.Q.L., met en évidence l'importance de la mise en œuvre, à l'égard des peuples autochtones du Québec, des droits économiques et sociaux reconnus sur le plan international. Le Pacte international relatif aux droits économiques sociaux et culturels reconnaît à tout individu le droit à la santé, le droit de ne pas faire l'objet de discrimination ou encore le droit à un niveau de vie suffisant. En particulier, son article 12 reconnait le droit qu'a toute personne de jouir du meilleur état de santé physique et mentale qu'elle soit capable d'atteindre. Ce droit comprend les mesures nécessaires pour diminuer la mortalité infantile, favoriser le développement sain des enfants, améliorer les aspects de l'hygiène du milieu, renforcer le traitement des maladies épidémiques et endémiques, créer les conditions propres à assurer

50. "If the various rituals are not performed according to the traditional rules, it is considered a moral offense, which will not only anger the spirit of the individual who died, but also may offend other ancestors of the community. This leads to a number of "spiritually-caused illnesses" that become manifest as actual physical maladies; however, they cannot be healed by conventional or Western means. These illnesses can potentially affect the entire natural lineage, that is, the descent group to which the deceased belonged. These problems and illnesses do not go away on their own, but must eventually be resolved through social and ceremonial means; if not, they will persist through generations ", Moiwana village $v$. Suriname, supra note 45 à la p. 26. 
à tous des services médicaux et une aide médicale en cas de maladie 51 .

À la différence de la Charte canadienne des droits et libertés, les droits économiques, sociaux et culturels sont reconnus au Québec par la Charte des droits et libertés de la personne52. Partant, ces droits sont intégrés à l'ordre juridique provincial, mais ne sont pas cependant contraignants ${ }^{53}$. Quant au droit à la santé en particulier, il n'est pas consacré par ce texte.

Néanmoins, les droits économiques, sociaux et culturels et les droits civils et politiques sont indivisibles par principe ${ }^{54}$. Or,

51. Pacte international relatif aux droits économiques, sociaux et culturels, article 12. Le Canada a ratifié les deux Pactes des Nations Unies le 19 mai 1976, en ligne: Nations Unies <http://www.unhchr.ch/ pdf/reportfr.pdf>.

52. Charte des droits et libertés de la personne, Chapitre IV, voir le texte de la Charte, en ligne : http://www.cdpdj.qc.ca/fr/commun/docs/charte.pdf.

53. Lucie Lamarche, "Le débat sur les droits sociaux au Canada respecte-t-il la juridicité de ces droits? ", dans Joel Bakan et David Schneiderman, dir., Social Justice and the Constitution, Ottawa, Carleton University Press, 1992 aux pp. 32 et 33; Pierre Bosset, "Les droits économiques et sociaux : parents pauvres de la Charte québécoise?" (1996) 75 R. du B. can. aux pp. 583-603; David Robitaille, "Les droits économiques et sociaux dans les relations État-particuliers après trente ans d'interprétation: normes juridiques ou énoncés juridiques symboliques? ", (2006) R. du B. hors série aux pp. 455-492.

54. Le principe de l'indivisibilité des droits vise à remettre en cause la hiérarchie établie entre droits civils et politiques et droits économiques et sociaux, les seconds étant essentiels pour assurer des conditions de vie minimales aux individus ainsi que pour garantir l'effectivité des droits civils et politiques. À ce titre, la Conférence mondiale sur les droits de l'homme indiquait déjà en 1993 que "Tous les droits de l'homme sont universels, indissociables, interdépendants et intimement liés ", Conférence mondiale sur les droits de l'homme, Déclaration et programme d'action de Vienne (12 juillet 1993), en ligne: <http://www.unhchr.ch/huridocda/huridoca.nsf/(Symbol)/A.CONF.157 .23.fr>au par. 5. La Fiche d'information no $16 \mathrm{du}$ Comité des droits économiques, sociaux et culturels explique également que "dans le droit international relatif aux droits de l'homme (comme dans son application au niveau national), les droits civils et politiques ont été privilégiés par rapport aux droits économiques, sociaux et culturels à bien des égards (moindre attention, codification du droit et interprétation judiciaire moins 
Le triptyque santé

- identités autochtones -

125

territoire. Perspectives juridiques

\section{l'absence de mise en œuvre des droits économiques et sociaux affecterait dans certains cas l'effectivité des droits civils et}

poussées et prise de conscience moins aiguë du public). On pense donc parfois à tort que seuls les droits civils et politiques (droit à un procès équitable, droit à l'égalité de traitement, droit à la vie, droit de vote, droit de ne pas être soumis à discrimination, etc.) peuvent faire l'objet de violations, de mesures de réparation et d'examen au regard du droit international. Les droits économiques, sociaux et culturels sont souvent perçus comme des droits de "seconde classe", qui ne sont pas obligatoires ni susceptibles de recours judiciaire et qui ne peuvent être assurés que "progressivement" avec le temps.

Cette manière de voir fait toutefois abstraction de la globalité du système des droits de l'homme, postulat déjà formulé en 1948 lors de l'adoption de la Déclaration universelle des droits de l'homme : l'indivisibilité et l'interdépendance des droits civils et politiques et des droits économiques, sociaux et culturels sont des principes fondamentaux du droit international relatif aux droits de l'homme qui ont été réaffirmés à maintes reprises, dont dernièrement en 1993 à la Conférence mondiale sur les droits de l'homme ", Comité des droits économiques, sociaux et culturels, Fiche d'information $n^{\circ} 16$ (Rev.1), (août 1996), en ligne <http://www.ohchr.org/Documents/Publications/FactSheet16Rev.1fr.pd f $>$ à la p. 3.

La Résolution 32/130 de l'Assemblée générale des Nations Unies va dans le même sens en indiquant que : " a) Tous les droits de l'homme et toutes les libertés fondamentales sont indivisibles et interdépendants; une attention égale et une considération urgente devront être accordées à la réalisation, la promotion et la protection tant des droits civils et politiques que des droits économiques, sociaux et culturels. b) La jouissance complète des droits civils et politiques est impossible sans celle des droits économiques, sociaux et culturels; les progrès durables dans la voie de l'application des droits de l'homme supposent une politique nationale et internationale rationnelle et efficace de développement économique et social, ainsi que le reconnaît la Proclamation de Téhéran de 1968 ", Assemblée générale des Nations Unies, Résolution 32/130 (16 décembre 1977), en ligne : Nations Unies <http://www.un.org/french/documents/view_doc.asp?symbol=A/RES/ 32/130\&Lang=F $>$ au par. 1 .

Voir aussi Secrétariat de l'Instance permanente des Nations Unies sur les questions autochtones, State of the World's Indigenous Peoples, ST/ESA/328, New York> à la p. 193 (2009), en ligne : United Nations $<$ http ://www.un.org/esa/socdev/unpfii/documents/SOWIP_web.pdf>. Asbjorn Eide, "Economic, Social and Cultural Rights as Human Rights ", dans Asbjorn Eide, Catarina Krause et Allan Rosas, dir., Economic, Social and Cultural Rights : A Textbook, 2e éd. , Boston, Martinus Nijhoff, 2001 aux pp. 9 et ss. 
politiques, rompant l'égalité entre la majorité et certaines populations marginalisées du Québec, telle que les Autochtones. En effet, le caractère non contraignant des droits économiques et sociaux pourrait avoir des conséquences plus grandes sur certaines populations, en raison de leur marginalisation, telles que l'absence d'accès à l'eau potable ou aux services de santé. Or, cette situation n'est pas favorable à l'exercice de droits civils et politiques - nous pensons par exemple au droit de vote ou au droit à la vie, à la liberté et à la sécurité protégés par la Charte canadienne des droits et libertés ${ }^{55}$. De ce fait, la relation de cause à effet, entre le respect des droits économiques, sociaux et cultures et le risque en santé, est confirmée en milieu autochtone, puisque le non respect de ces droits entretient le risque sanitaire.

Ainsi, la production du droit et sa mise en œuvre ne sont pas neutres. Elles sont souvent déterminées par les besoins, conceptions et modèles majoritaires, ce qui a pour conséquence d'entretenir le risque en santé. En effet, l'élaboration et l'application du droit ont tendance à négliger les identités autochtones et à ignorer leur interconnexion avec la santé et l'accès au territoire. En outre, la hiérarchie des droits priorisée par les autorités peut avoir pour effet de rompre l'égalité entre les citoyens d'un même État, la valeur des droits économiques et sociaux au Québec et au Canada le démontre. De ce fait, la nécessité de travailler à une transformation du droit interne et des conceptions qui l'imprègnent est nécessaire, par exemple en ce qui a trait à l'interprétation du principe d'égalité tel que proposé par la Charte canadienne des droits et libertés.

\section{Conclusion}

En contexte autochtone, santé, identité, droit au territoire et aux ressources naturelles forment un tout interrelié. Cette équation, bien que présentée au sein de la Province du Québec,

55. Charte canadienne des droits et libertés, partie I de la Loi constitutionnelle de 1982, constituant l'annexe $\mathrm{B}$ de la Loi de 1982 sur le Canada (R.U.).1982, c. 11 , art. 3, 7 . 
$\begin{array}{cc}\text { (2009-10) } 40 \text { R.D.U.S. } & \text { Le triptyque santé } \\ \text { - identités autochtones - } & \text { territoire. Perspectives juridiques }\end{array}$

est, a priori, transférable pour l'ensemble des peuples autochtones.

Le fait de regarder la problématique autochtone sous l'angle du risque en santé permet de souligner et de renforcer l'importance de l'identité et du territoire. Cet angle d'approche dispose d'un intérêt majeur, puisqu'il met en exergue l'étendue des dangers dus aux négligences actuelles et l'urgence d'un changement de considération et de vision plus profond, quant à la manière d'aborder la question autochtone. Ce changement doit se produire au niveau politique et social. Il implique également des mutations juridiques, à savoir un changement en termes de regard porté sur la question autochtone (comment l'aborder juridiquement?) et une transformation dans la définition même du droit (qu'est-ce que le droit lorsque nous nous situons dans une perspective autochtone?).

Or, le droit tel qu'il est élaboré et appliqué actuellement n'est pas toujours adapté. De ce fait, il reproduit les schémas politiques et sociaux d'exclusion. Le droit pourrait contribuer à la réduction du risque en santé chez les peuples autochtones, si les schémas politiques et sociaux se transformaient et si le désir d'accommodement et de refondation d'un projet de société commun aux Autochtones et aux non-Autochtones voyait le jour. Dans ce contexte, la réconciliation, passant par le dialogue, la reconnaissance de la colonisation et de ses effets, est essentielle ${ }^{56}$. En ce sens, la réalité contemporaine du risque en santé, au sein des communautés autochtones du Québec, nous ramène une fois encore à la relativité du droit. Sa transformation dépend de ceux qui ont le pouvoir de le produire et de le sanctionner ${ }^{57}$, c'est-à-dire des acteurs politiques et sociaux.

56. Denise C. Breton, "Digging Deeper: Challenges for Restorative Justice ", dans Wanda D. McCaslin, supra note 23 aux pp. 409-434.

57. Parmi eux se trouvent les communautés autochtones et leurs représentants, qui ne cessent de déployer leurs énergies en négociant avec les différents paliers de gouvernements, en revendiquant et en s'affirmant. Par ailleurs, l'État et ses institutions sont des acteurs puissants. Ils sont davantage représentatifs de la majorité non- 
Bien qu'elle ne soit pas achevée et qu'elle ne se développe pas identiquement en droit interne et en droit international, cette transformation est actuellement à l'œuvre. En droit supranational, les décisions de la Cour interaméricaine des droits de l'homme ainsi que l'adoption de la Déclaration des Nations sur les droits des peuples autochtones par 145 États 58 , y contribuent. Elle passe premièrement par la reconnaissance de différentes normes juridiques, telles que le droit de propriété collective des territoires ancestraux $^{59}$ et les droits aux territoires et aux ressources ${ }^{60}$, le droit au consentement préalable, libre et éclairé61, le droit au respect des modes de vie autochtones ${ }^{62}$, le droit d'association à

autochtone et conservent une grande puissance dans la détermination des normes, puisqu'ils les sanctionnent. Entre les deux se situent l'ensemble des acteurs non-étatiques (lobbying, militants, membres de la société civile, compagnies d'exploitation minières, forestières, etc.) qui exercent une pression fondamentale sur l'orientation des normes juridiques en fonction de leurs intérêts et idéaux propres, favorables ou non à une transformation du droit prenant en compte le point de vue des peuples autochtones.

58. La Déclaration des Nations Unies sur les droits des peuples autochtones a été signée par 144 États le 13 septembre 2007 à l'Assemblée générale des Nations Unies. Au total, 11 États se sont abstenus et 4 ont voté contre, à savoir les États-Unis, la Nouvelle-Zélande, l'Australie et le Canada. Depuis, l'Australie est revenue sur sa position et a adopté le texte le 03 avril 2009. Voir en ligne: Nations Unies : <http://www.un.org/esa/ socdev/unpfii/fr/drip.html>.

59. Nicaragua, supra note 49 ; Suriname, supra note 45; Comunidad Indigena Yakye Axa $v$. Paraguay, requête no 12313, arrêt, (17 juin 2005) en ligne : CIDH <http : //www.corteidh.or.cr/docs/casos/articulos/seriec_125_ ing.pdf $>$.

60. Déclaration des Nations Unies sur les droits des peuples autochtones, art. 26. En ce qui concerne le droit aux ressources naturelles en contexte interaméricain, voir la décision Saramaka People $v$. Suriname, requête $\mathrm{n}^{\circ}$ 12338, arrêt, (28 novembre 2007) en ligne: C.I.D.H. <http://www. corteidh.or.cr/docs/casos/articulos/seriec_172_ing.pdf $>$ [Saramaka People].

61. Déclaration des Nations Unies sur les droits des peuples autochtones, art. 28. Au sein du système interaméricain, voir aussi la décision Saramaka People, supra note 60.

62. Cette norme juridique émerge actuellement, pour les peuples autochtones, au sein des systèmes juridiques européen, interaméricain et onusien. Elle provient de l'interprétation évolutive et adaptée par les juges supranationaux des Conventions internationales sur les droits de 
$\begin{array}{cc}\text { (2009-10) } 40 \text { R.D.U.S. } & \text { Le triptyque santé } \\ \text { - identités autochtones - } & \text { territoire. Perspectives juridiques }\end{array}$

l'élaboration et à la définition des programmes de santé63 et le droit à leurs propres pratiques médicales ${ }^{64}$. La relation particulière des peuples autochtones avec leurs territoires ancestraux est également consacrée. Cela peut être favorable à la diminution du risque en santé. En effet, les droits aux territoires et aux ressources, d'une part, facilitent l'accès à la pratique de la médecine traditionnelle65 et peuvent permettre une autodétermination. D’autre part, en rétablissant la dignité de ces peuples, les droits aux territoires pourraient diminuer le malaise identitaire, source de risque en santé. Ces normes représentent donc une reconnaissance juridique supranationale des identités autochtones, qui n'a cependant pas toujours de conséquence immédiate en droit interne ${ }^{66}$. Deuxièmement, la transformation du droit se manifeste également par l'association des peuples autochtones à l'élaboration des normes les concernant. Ce fut le cas, au niveau international, pour la rédaction de la Déclaration des Nations Unies sur le droit des peuples autochtones. C'est aussi le cas, actuellement, pour l'élaboration du projet de Déclaration américaine relative aux droits des peuples autochtones et pour le projet de Convention nordique samie. Une forme d'association se produit également sur le plan interne en matière de gestion des services de santé, si l'on en croit les diverses ententes conclues. L'association des bénéficiaires des normes à leur détermination est un instrument essentiel à l'adoption de règles adéquates. On peut donc constater que le rôle du droit dans la diminution du

l'homme, telles que les conventions européenne et américaine des droits de l'homme ainsi que le Pacte international relatif aux droits civils et politiques. Cette norme est prétorienne en ce qu'elle se fonde sur diverses décisions et communications.

63. Déclaration des Nations Unies sur les droits des peuples autochtones, art. 23.

64. Ibid., art. 24.

65. Les droits au territoire ainsi que la reconnaissance identitaire devraient permettre, d'une part, un accès aux ressources naturelles qui sont à la base de la médecine traditionnelle. Ils devraient permettre, ensuite, une certaines autonomisation ou du moins une coopération dans la gestion des services de santé. Sur ce point, voir supra note 34.

66. En effet, le Canada n'a pas signé la Déclaration des Nations Unies sur les droits des peuples autochtones et n'a pas reconnu la juridiction de la Cour interaméricaine des droits de l'homme. 
risque en santé chez les peuples autochtones ne passe pas uniquement par la réglementation en santé, mais davantage par l'acquisition de perspectives juridiques plus globales. Le processus de prise de pouvoir par les Autochtones eux-mêmes est une nécessité inhérente à cette transformation. Il va de pair avec le processus de reconnaissance sur le plan politique et la mise en œuvre du principe d'autodétermination en matière juridique. Néanmoins, cette prise de pouvoir ne peut aboutir seule et doit être accompagnée d'une volonté sociale de transformation à l'échelle de la société toute entière, puisque les Autochtones ne peuvent maitriser seul les déterminismes sociaux conduisant à l'exclusion ou à la pauvreté. De plus, elle ne peut aboutir sans l'élaboration d'objectifs et de modalités réalistes de participation.

Enfin, la mutation des ordres juridiques n'est pas autonome, dans la mesure où elle dépend de la transformation des perspectives politiques et sociales. La méconnaissance des identités autochtones, la rigidité des normes juridiques internes, leur cloisonnement encore à l'œuvre et le manque de considération au regard des revendications autochtones par les États y sont autant d'obstacles 67 .

Ainsi, la situation de non-reconnaissance, de pauvreté, d'exclusion et de discrimination, sources du risque en santé pour les peuples autochtones du Québec, a tendance à se perpétuer, sous la bienveillance d'un droit dépendant des intérêts sociopolitiques privilégiés. En ce sens, le grand principe de dignité, garanti à tous par de nombreuses conventions supranationales ainsi que par le droit canadien interne, tant fédéral que provincial, paraît être vidé de sa substance.

67. L'avènement d'un droit au respect des modes de vie autochtones pourrait, néanmoins, représenter un pas supplémentaire, puisqu'à la différence des autres normes déjà consacrées, il dispose d'une capacité englobante. 